\title{
A SYMMETRIC DIVERGENCE MEASURE AND ITS BOUNDS
}

\author{
K. C. JAIN AND RUCHI MATHUR
}

\begin{abstract}
A new symmetric divergence measure is proposed which is useful in comparing two probability distributions. This non-parametric measure belongs to the Csiszar's $f$ divergence class. Its properties are studied and bounds are obtained in terms of some well known divergence measures. A numerical illustration based on the probability distribution is carried out.
\end{abstract}

\section{Introduction}

One problem in Probability theory which researchers has been trying to find is an appropriate measure of divergence (or distance or difference or discrimination or information) between two probability distributions. Non parametric measure give the amount of information supplied by the data for discriminating in favour of a probability distribution against another or for measuring the distance or affinity between two probability distributions. There are a number of divergence measures being proposed in literature which compare two probability distributions and have been applied in a variety of disciplines such as antropology, genetics, finance, economics and political science, biology, analysis of contingency of tables, approximations of probability distributions, signal processing and pattern recognition.

In this paper we present a non-parametric symmetric divergence measure which belongs to the class of Csiszar's $f$ divergences $(4,5)$. In Section 2 we discuss the Csiszar's $f$ divergences and inequalities. New symmetric divergence measure is obtained in Section 3. In Section 4 we have derived some inequalities providing bounds for the new measure in terms of some well known divergence measures. The parametric measure of information obtained from the suggested non- parametric divergence is given in Section 5. The suggested measure is compared with other measures in Section 6.

Corresponding author: Ruchi Mathur. 2010 Mathematics Subject Classification. 94A17, $26 \mathrm{D} 15$.

Key words and phrases. Symmetric, Csiszar's f divergence, parametric measure. 


\section{Csiszar's $f$-divergence and inequalities}

Let $\Omega=\left\{x_{1}, x_{2}, \ldots\right\}$ be a set with at least two elements and $\mathbb{P}$ the set of all probability distribution $P=(p(x): x \in \Omega\}$. For a convex function $f:(0, \infty) \rightarrow \mathbb{R}$, the $f$-divergence of the probability distributions $P$ and $Q$ by Csiszar, $(4,5)$ and Ali \& Silvey [1] is defined as

$$
C_{f}(P, Q)=\sum_{x \in \Omega} q(x) f\left(\frac{p(x)}{q(x)}\right) .
$$

Henceforth, for brevity we will denote $C_{f}(P, Q), p(x)$ and $\sum_{x \in \Omega}$ by $C(P, Q), p, q$ and $\sum$ respectively.

Osterreicher [19] has discussed basic general properties of $f$-divergences including their axiomatic properties and some important classes. During the recent past, there has been a considerable amount of work providing different kind of bounds on the distance, information and divergence measures [7, 8, 9, 27]. Taneja and Kumar [24] unified and generalized three theorems studied by Dragomir $[7,8,9]$ which provide bounds on $C(P, Q)$. The main result in [24] is the following theorem:

Theorem 2.1. Let $f: I \subset \mathbb{R}_{+} \rightarrow \mathbb{R}$ be a mapping which is normalized, i.e., $f(1)=0$ and suppose that

(i) $f$ is twice differentiable on $(r, R), 0 \leq r \leq 1 \leq R<\infty$, ( $f^{\prime}$ and $f^{\prime \prime}$ denote the first and second derivatives of $f$ ),

(ii) there exist real constants $m, M$ such that $m<M$ and $m<x^{2-s} f^{\prime}(x)<M, \forall x \in(r, R)$, $s \in \mathbb{R}$.

if $P, Q \in \mathbb{P}^{2}$ are discrete probability distributions with $0<r \leq \frac{p}{q} \leq R \leq \infty$, then

$$
m \Phi_{s}(P, Q) \leq C(P, Q) \leq M \Phi_{s}(P, Q)
$$

and

$$
m\left(\eta_{s}(P, Q)-\Phi_{s}(P, Q)\right) \leq C_{\rho}(P, Q)-C(P, Q) \leq M\left(\eta_{s}(P, Q)-\Phi_{s}(P, Q)\right)
$$

where

$$
\begin{aligned}
\Phi_{s}(P, Q) & = \begin{cases}{ }^{2} K_{s}(P, Q), & s \neq 0,1 \\
K(Q, P), & s=0 \\
K(P, Q), & s=1\end{cases} \\
{ }^{2} K_{s}(P, Q) & =[s(s-1)]^{-1}\left[\sum p^{s} q^{1-s}-1\right], \quad s \neq 0,1, \\
k(P, Q) & =\sum p \ln \left(\frac{p}{q}\right), \\
C_{\rho}(P, Q) & =C_{f}\left(\frac{P^{2}}{Q}, P\right)-C_{f}(P, Q)=\sum(p-q) f\left(\frac{p}{q}\right),
\end{aligned}
$$


and

$$
\begin{aligned}
\eta_{s}(P, Q) & =C_{\phi^{\prime} s}\left(\frac{P^{2}}{Q}, P\right)-C_{\phi^{\prime} s}(P, Q) \\
& = \begin{cases}(s-1)^{-1} \sum(p-q)\left(\frac{p}{q}\right)^{s-1}, & s \neq 1 \\
\sum(p-q) \ln \left(\frac{p}{q}\right), & s=1 .\end{cases}
\end{aligned}
$$

The following information inequalities which are interesting from the information-theoretic point of view, are obtained from Theorem 2.1 and discussed in [24]

(i) The case $s=2$ provides the information bounds in terms of the Chi-square divegence $\chi^{2}(P, Q)$ :

$$
\frac{m}{2} \chi^{2}(P, Q) \leq C(P, Q) \leq \frac{M}{2} \chi^{2}(P, Q)
$$

and

$$
\frac{m}{2} \chi^{2}(P, Q) \leq C_{\rho}(P, Q)-C(P, Q) \leq \frac{M}{2} \chi^{2}(P, Q)
$$

where

$$
\chi^{2}(P, Q)=\sum \frac{(p-q)^{2}}{q} .
$$

(ii) For $s=1$, the information bounds in terms of the Kullback-Leibler divergence, $K(P, Q)$ :

$$
m K(P, Q) \leq C(P, Q) \leq M K(P, Q)
$$

and

$$
m K(Q, P) \leq C_{\rho}(P, Q)-C(P, Q) \leq M K(Q, P) .
$$

(iii) The case $s=\frac{1}{2}$ provides the information bounds in terms of the Hellinger's descrimination, $h(P, Q)$ :

$$
4 m h(P, Q) \leq C(P, Q) \leq 4 M h(P, Q)
$$

and

where

$$
\begin{aligned}
4 m\left(\frac{1}{4} \eta_{1 / 2}(P, Q)-h(P, Q)\right) & \leq C_{\rho}(P, Q)-C(P, Q) \\
& \leq 4 M\left(\frac{1}{4} \eta_{1 / 2}(P, Q)-h(P, Q)\right)
\end{aligned}
$$

$$
h(P, Q)=\sum \frac{(\sqrt{p}-\sqrt{q})^{2}}{2} .
$$

(iv) for $s=0$, the information bounds in terms of the Kullback- Leibler and $\chi^{2}$-divergences:

$$
m K(P, Q) \leq C(P, Q) \leq M K(P, Q),
$$

and

$$
m\left(\chi^{2}(Q, P)-K(Q, P)\right) \leq C_{\rho}(P, Q)-C(P, Q) \leq M\left(\chi^{2}(Q, P)-K(Q, P)\right) .
$$




\section{A symmetric divergence measure of the Csiszar's $f$-divergence family}

We consider the function $f:(0, \infty) \rightarrow \mathbb{R}$

$$
\text { given by } f(u)=\frac{(u-1)^{4}(u+1)\left(u^{2}+1\right)}{u^{3}}
$$

and thus the divergence measure.

$$
P^{*}(P, Q) ; C_{f}(P, Q)=\frac{(p-q)^{4}(p+q)\left(p^{2}+q^{2}\right)}{p^{3} q^{3}} .
$$

Since

$$
f^{\prime}(U)=\frac{(u-1)^{3}\left[4 u^{4}+3 u^{3}+3 u^{2}+3 u+3\right]}{u^{4}}
$$

and

$$
f^{\prime}(u)=\frac{(u-1)^{2}}{u^{5}}\left[12 u^{5}+6 u^{4}+6 u^{3}+6 u^{2}+6 u+12\right] .
$$

It follows that $f^{\prime \prime}(u)>0$ for all $u>0$.

Hence $f(u)$ is convex for all $u>0$.

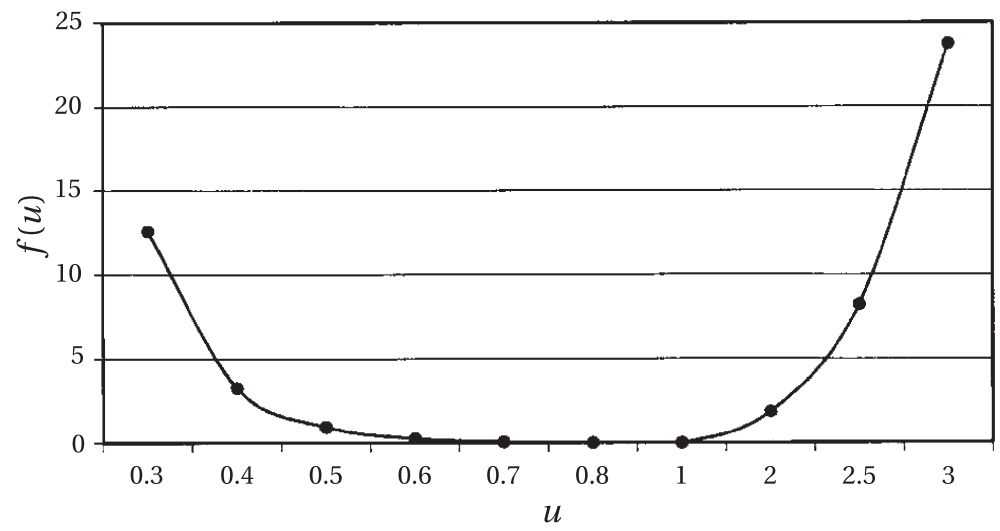

Figure 3.1: Graph of the Convex Function $f(u)$.

Further $f(1)=0$. Thus we can say that the measure is non negative and convex in the pair of probability distribution $(P, Q) \in \Omega$.

\section{Bounds for $P^{*}(P, Q)$}

In this section, we will derive information divergence inequalities providing bounds for $P^{*}(P, Q)$ in terms of some well known divergence measures. We start by deriving bounds in terms of the Chi-Square divergence $\chi^{2}(P, Q)$. 
Proposition 4.1. Let $\chi^{2}(P, Q$,$) and P^{*}(P, Q)$ be defined as in (2.11) and (3.2) respectively. For $P, Q \in \mathbb{P}^{2}$ and $0<r \leq \frac{p}{q} \leq R<\infty$ we have

$$
\begin{aligned}
& \frac{3(R-1)^{2}}{R^{5}}\left[2 R^{5}+R^{4}+R^{3}+R^{2}+R+2\right] \chi^{2}(P, Q) \leq P^{*}(P, Q) \\
& \leq \frac{3(r-1)^{2}}{r^{5}}\left[2 r^{5}+r^{4}+r^{3}+r^{2}+r+2\right] \chi^{2}(P, Q)
\end{aligned}
$$

and

where

$$
\begin{aligned}
& \frac{3(R-1)^{2}}{R^{5}}\left[2 R^{5}+R^{4}+R^{3}+R^{2}+R+2\right] \chi^{2}(P, Q) \leq P_{\rho}^{*}(P, Q)-P^{*}(P, Q) \\
& \quad \leq \frac{3(r-1)^{2}}{r^{5}}\left[2 r^{5}+r^{4}+r^{3}+r^{2}+r+2\right] \chi^{2}(P, Q)
\end{aligned}
$$

$$
P_{\rho}^{*}(P, Q)=\frac{(p-q)\left(p^{3}+q^{3}\right)\left(4 p^{4}+3 p^{3} q+3 p^{2} q^{2}+3 p q^{3}+3 q^{3}\right)}{p^{4} q^{3}}
$$

Proof. From the function $f(u)$ in (3.1) we have

$$
f^{\prime}(u)=\frac{(u-1)^{3}\left[4 u^{4}+3 u^{3}+3 u^{2}+3 u+3\right]}{u^{4}} .
$$

And thus the

$$
\begin{aligned}
P_{\rho}^{*}(P, Q) & =\sum(p-q) f^{\prime}\left(\frac{p}{q}\right) \\
& =\frac{(p-q)\left(p^{3}+q^{3}\right)\left(4 p^{4}+3 p^{3} q+3 p^{2} q^{2}+3 p q^{3}+3 q^{3}\right)}{p^{4} q^{3}}
\end{aligned}
$$

Further

$$
f^{\prime \prime}(u)=\frac{6(u-1)^{2}}{u^{5}}\left[2 u^{5}+u^{4}+u^{3}+u^{2}+u+2\right] .
$$

Now if $u \in[a, b] \subset(0, \infty)$ then

or accordingly

$$
\begin{gathered}
\frac{6(b-1)^{2}}{b^{5}}\left[2 b^{5}+b^{4}+b^{3}+b^{2}+b+2\right] \leq f^{\prime \prime}(u) \\
\leq \frac{6(a-1)^{2}}{a^{5}}\left[2 a^{5}+a^{4}+a^{3}+a^{2}+a+2\right]
\end{gathered}
$$

$$
\begin{aligned}
& \frac{6(R-1)^{2}}{R^{5}}\left[2 R^{5}+R^{4}+R^{3}+R^{2}+R+2\right] \leq f^{\prime \prime}(u) \\
& \leq \frac{6(r-1)^{2}}{r^{5}}\left[2 r^{5}+r^{4}+r^{3}+r^{2}+r+2\right]
\end{aligned}
$$

where $r$ and $R$ are defined above.

Thus in view of (2.9) and (2.10) we get inequalities (4.1) and (4.2). The information bounds in terms of Kullback - Leibler divergence $K(P, Q)$ follows. 
Proposition 4.2. Let $K(P, Q), P^{*}(P, Q)$ and $P_{\rho}^{*}(P, Q)$ be defined as (2.6), (3.2) and (4.3) respectively.

For $P, Q, \in \mathbb{P}^{2}$ and $0<r \leq \frac{p}{q} \leq R \leq \infty$ the

$$
\begin{aligned}
& \frac{6(R-1)^{2}}{R^{5}}\left[2 R^{5}+R^{4}+R^{3}+R^{2}+R+2\right] K(P, Q) \leq P^{*}(P, Q) \\
& \quad \leq \frac{6(r-1)^{2}}{r^{5}}\left[2 r^{5}+r^{4}+r^{3}+r^{2}+r+2\right] K(P, Q)
\end{aligned}
$$

and

$$
\begin{aligned}
& \frac{6(R-1)^{2}}{R^{5}}\left[2 R^{5}+R^{4}+R^{3}+R^{2}+R+2\right] K(P, Q) \leq P^{*}(P, Q)-P_{\rho}^{*}(P, Q) \\
& \quad \leq \frac{6(r-1)^{2}}{r^{5}}\left[2 r^{5}+r^{4}+r^{3}+r^{2}+r+2\right] K(P, Q) .
\end{aligned}
$$

Proof. from (3.4) $f^{\prime \prime}(u)=\frac{6(u-1)^{2}}{u^{5}}\left[2 u^{5}+u^{4}+u^{3}+u^{2}+u+2\right]$. Let the function $g:[r, R] \rightarrow \mathbb{R}$ be Such that

$$
\begin{aligned}
g(u) & =u f^{\prime \prime}(u)=\frac{6(u-1)^{2}}{u^{4}}\left[2 u^{5}+u^{4}+u^{3}+u^{2}+u+2\right] \text { then } \\
\inf _{u \in(r, R)} g(u) & =\frac{6(R-1)^{2}}{R^{4}}\left[2 R^{5}+R^{4}+R^{3}+R^{2}+R+2\right]
\end{aligned}
$$

and

$$
\sup _{u \in(r, R)} g(u)=\frac{6(r-1)^{2}}{r^{4}}\left[2 r^{5}+r^{4}+r^{3}+r^{2}+r+2\right] .
$$

The inequalities (4.9) and (4.10) follow from (2.12) and (2.13) using (4.12) and (4.13).

The following proposition provides the information bounds in terms of the Hellinger's discrimination $h(P, Q)$ and $\eta_{1 / 2}(P, Q)$.

Proposition 4.3. Let $_{1 / 2}(P, Q), h(P, Q), P^{*}(P, Q)$ and $P_{\rho}^{*}(P, Q)$ be defined as in (2.7), (2.15), (3.2) and (4.5) respectively. For $P, Q \in \mathbb{P}^{2}$ and and $0<r \leq \frac{p}{q} \leq R<\infty$

$$
\begin{gathered}
\frac{24(r-1)^{2}\left[2 r^{5}+r^{4}+r^{3}+r^{2}+r+2\right] h(P, Q)}{r^{5}} \leq P^{*}(P, Q) \\
\leq \frac{24(R-1)^{2}}{R^{5}}\left[2 R^{5}+R^{4}+R^{3}+R^{2}+R+2\right] h(P, Q)
\end{gathered}
$$

and

$$
\begin{aligned}
& \frac{24(r-1)^{2}}{r^{5}}\left[2 r^{5}+r^{4}+r^{3}+r^{2}+r+2\right]\left(\frac{1}{4} \eta_{1 / 2}(P, Q)-h(P, Q)\right. \\
& \left.\quad \leq P_{\rho}^{*}(P, Q)-P^{*}(P, Q) \leq \frac{24(R-1)^{2}}{R^{5}}\left[2 R^{5}+R^{4}+R^{3}+R^{2}+R+2\right]\right) \\
& \quad\left(\frac{1}{4} \eta_{1 / 2}(P, Q)-h(P, Q)\right)
\end{aligned}
$$


Proof. We have $f^{\prime \prime}(u)=\frac{6(u-1)^{2}}{u^{5}}\left[2 u^{5}+u^{4}+u^{3}+u^{2}+u+2\right]$ from (3.4). Let the function $g=$ $[r, R] \rightarrow \mathbb{R}$ be such that

$$
g(u)=u^{3 / 2} f^{\prime \prime}(u)=\frac{6(u-1)^{2}\left[2 u^{5}+u^{4}+u^{3}+u^{2}+u+2\right]}{u^{7 / 2}}
$$

then

and

$$
\inf _{u \in(r, R)} g(u)=\frac{6(r-1)^{2}\left[2 r^{5}+r^{4}+r^{3}+r^{2}+r+2\right]}{u^{7 / 2}}
$$

$$
\sup _{u \in(r, R)} g(u)=\frac{6(R-1)^{2}\left[2 R^{5}+R^{4}+R^{3}+R^{2}+R+2\right]}{R^{7 / 2}} .
$$

Thus the inequalities (4.14) and (4.15) are established using (2.14), (2.15), (4.17) and (4.18).

Proposition 4.4. Let $K(P, Q), \chi^{2}(P, Q), P^{*}(P, Q)$ and $P_{\rho}^{*}(P, Q)$ be defined as in (2.5), (2.10), (3.2) and (4.5) respectively.

$$
\begin{aligned}
& \text { If }(P, Q) \in \mathbb{P}^{2} \text { and } 0<r \leq \frac{p}{q} \leq R<\infty \text {, then } \\
& \qquad \begin{array}{l}
\frac{6(r-1)^{2}}{r^{5}}\left[2 r^{5}+r^{4}+r^{3}+r^{2}+r+2\right] K(P, Q) \leq P^{*}(P, Q) \\
\leq \frac{6(R-1)^{2}}{R^{5}}\left[2 R^{5}+R^{4}+R^{3}+R^{2}+R+2\right] K(P, Q)
\end{array}
\end{aligned}
$$

and

$$
\begin{aligned}
& \frac{6(r-1)^{2}}{r^{5}}\left[2 r^{5}+r^{4}+r^{3}+r^{2}+r+2\right]\left[\chi^{2}(Q, P)-K(Q, P)\right] \\
& \quad \leq\left[P_{\rho}^{*}(P, Q)-P^{*}(P, Q)\right] \\
& \quad \frac{6(R-1)^{2}}{R^{5}}\left[2 R^{5}+R^{4}+R^{3}+R^{2}+R+2\right]\left[\chi^{2}(Q, P)-K(Q, P)\right]
\end{aligned}
$$

Proof. From (3.4) $f^{\prime \prime}(u)=\frac{6(u-1)^{2}}{u^{5}}\left[2 u^{5}+u^{4}+u^{3}+u^{2}+u+2\right]$.

Let the function $g:(r, R) \rightarrow \mathbb{R}$ be such that

$$
g(u)=u^{2} f^{\prime \prime}(u)=\frac{6(u-1)^{2}\left[2 u^{5}+u^{4}+u^{3}+u^{2}+u+2\right]}{u^{3}}
$$

then

$$
\inf _{u \in(r, R)} g(u)=\frac{6(r-1)^{2}}{r^{3}}\left[2 r^{5}+r^{4}+r^{3}+r^{2}+r+2\right]
$$

and

$$
\sup _{u \in(r, R)} g(u)=\frac{6(R-1)^{2}}{R^{3}}\left[2 R^{5}+R^{4}+R^{3}+R^{2}+R+2\right] .
$$

Thus (4.19) and (4.20) follows from (2.17) and (2.18) using (4.21) and (4.22). 


\section{Parametric measure of information $P^{*^{c}}(P, Q)$}

The Parametric measures of information are applicable to regular families of probability distributions, that is, to the families for which the following regularity conditions are assumed to be satisfied. Let for $\boldsymbol{\theta}=\left(\theta_{1}, \ldots, \theta_{k}\right)$, the Fisher [16] information matrix be

$$
I_{X}(\theta)= \begin{cases}E_{\theta}\left[\frac{\partial}{\partial \theta} \log f(X, \theta)\right]^{2}, & \text { if } \theta \text { is univariate; } \\ \|\left. E_{\theta}\left[\frac{\partial}{\partial \theta_{i}} \log f(X, \theta) \frac{\partial}{\partial \theta_{j}} \log f(X, \theta)\right]\right|_{k \times k} & \text { if } \theta \text { is } k \text {-variate; }\end{cases}
$$

where $\|\cdot\|$ denotes a $k \times k$ matrix.

The regularity conditions are:

(R1) $f(x, \theta)<0$ for all $x \in \Omega$ and $\theta \in \Theta$;

(R2) $\frac{\partial}{\partial \theta}, f(x)$ exists for all $x \in \Omega$ and $\theta \in \Theta$; and all $i=1, \ldots, k$;

(R3) $\frac{\partial}{d \theta_{i}} \int_{A} f(x, \theta) d \mu=\int_{A} \frac{d}{d \theta_{j}}(x, \theta) d \mu$ for any $A \in A$ (measurable space $(X, A$ in respect of a finite or $\sigma$-finite measure $\mu$ ), all $\theta \in$ and all $i$ in respect.

Ferentimos and Papaioannou [13] suggested the following method to construct the parametric measure from the non-parametric measure:

Let $k(A)$ be a one-to-one transformation of the parameter space $\Theta$ onto itself with $k(\theta) \neq$ $\theta$ the quantity

$$
\operatorname{Ix}[\theta, k(\theta)]=\operatorname{Ix}[f(x, \theta), f(x, k(\theta))],
$$

can be considered as parametric measure of information based on $k(\theta)$. This method is employed to construct the modified Csiszar's measure of information about univariate $\theta$ contained in $X$ and based on $k(\theta)$ as

$$
I_{x}^{C}[\theta, k(\theta)]=\int f(x, \theta), \phi\left(\frac{f(x, k(\theta))}{f(x, \theta)}\right) d \mu .
$$

Now we have the following proposition for providing the parameteric measure of information from $P^{*}(P, Q)$.

Proposition 5.1. Let the convex function $\phi:(0, \infty) \rightarrow \mathbb{R}$ be

$$
\Phi(u)=\frac{(u-1)^{2}(u+1)\left(u^{2}+1\right)}{u^{3}} .
$$

and corresponding non-parametric divergence measure

$$
P^{*}(P, Q)=\frac{\sum(p-q)^{4}(p+q)\left(p^{2}+q^{2}\right)}{p^{3} q^{3}} .
$$

Then the parametric measure $P^{* c}(P, Q)$

$$
P^{*^{c}}(P, Q)=I_{x}^{c}[\theta, K(\theta)]=\frac{\sum(p-q)^{4}(p+q)\left(p^{2}+q^{2}\right)}{p^{3} q^{3}} .
$$


Proof. For discrete random variables $X$, the expression (5.3) can be written as

$$
I_{x}^{c}[\theta, K(\theta)]=\sum_{x \in \Omega} p(x) \phi\left[\frac{q(x)}{p(x)}\right]
$$

from (5.4) we have

$$
\phi\left[\frac{q(x)}{p(x)}\right]=\frac{(p-q)^{4}(p+q)\left(p^{2}+q^{2}\right)}{p^{4} q^{3}}
$$

where we denote $p(x)$ and $q(x)$ by $p$ and $q$ respectively. Thus $P^{*^{c}}(P, Q)$ in (5.5) follows from (5.6) and (5.7). The parametric measure $P^{*^{c}}(P, Q)$ is the same as the non-parametric measure $P^{*}(P, Q)$. Further since the properties of $P^{*}(P, Q)$ do not require any regularity conditions, $P^{*}(P, Q)$ is applicable to the broad family of probability distribution including the non-regular ones.

\section{Numerical illustration}

We consider an example of symmetrical probability distribution. We calculate measures $\Psi(P, Q), \chi^{2}(P, Q), J(P, Q)$ and $P^{*}(P, Q)$ and compare bounds. Here $J(P, Q)=K(P, Q)+K(Q, P)=$ $\sum(p-q) \ln \left(\frac{p}{q}\right)$ is the Kullback Leibler divergence.

Example 6.1. Let $P$ be the binomial probability distribution for the random valuable $X$ with parameter $(n=8, p=0.5)$ and $Q$ its approximated normal probability distribution.

Table 1: Binomial Probability Distribution.

\begin{tabular}{|c|c|c|c|c|c|c|c|c|c|}
\hline$x$ & 0 & 1 & 2 & 3 & 4 & 5 & 6 & 7 & 8 \\
\hline$p(x)$ & 0.004 & 0.031 & 0.109 & 0.219 & 0.274 & 0.219 & 0.109 & 0.031 & 0.004 \\
$q(x)$ & 0.005 & 0.030 & 0.104 & 0.220 & 0.282 & 0.220 & 0.104 & 0.030 & 0.005 \\
$\frac{p(x)}{q(x)}$ & 0.774 & 1.042 & 1.0503 & 0.997 & 0.968 & 0.997 & 1.0503 & 1.042 & 0.774 \\
\hline
\end{tabular}

The measure $\Psi(P, Q), \chi^{2}(P, Q), J(P, Q)$ and $P *(P, Q)$ are

$$
\begin{array}{rr}
\Phi(P, Q)=0.00305063 & J(P, Q)=0.00151848 \\
\chi^{2}(P, Q)=0.00145837 & P^{*}(P, Q)=0.00009743 .
\end{array}
$$

It is noted that

$$
r=(0.774179933) \leq \frac{p}{q} \leq R(1.050330018) .
$$

The lower and upper bounds for $P^{*}(P, Q)$ from (4.1)

$$
\text { Lower Bound }=\frac{3(R-1)^{2}}{R^{5}}[2 R 5+R 4+R 3+R Z+R+2] \chi^{2}(P, Q)=0.0000783124
$$




$$
\text { Upper Bound }=\frac{3(r-1)^{2}}{r^{5}}\left[2 r^{5}+r^{4}+r^{3}+r^{2}+r+2\right] \chi^{2}(P, Q)=0.00106015
$$

and thus $0.0000783124<P^{*}(P, Q)=0.00009743<0.00106015$. The width of the interval is 0.00098184

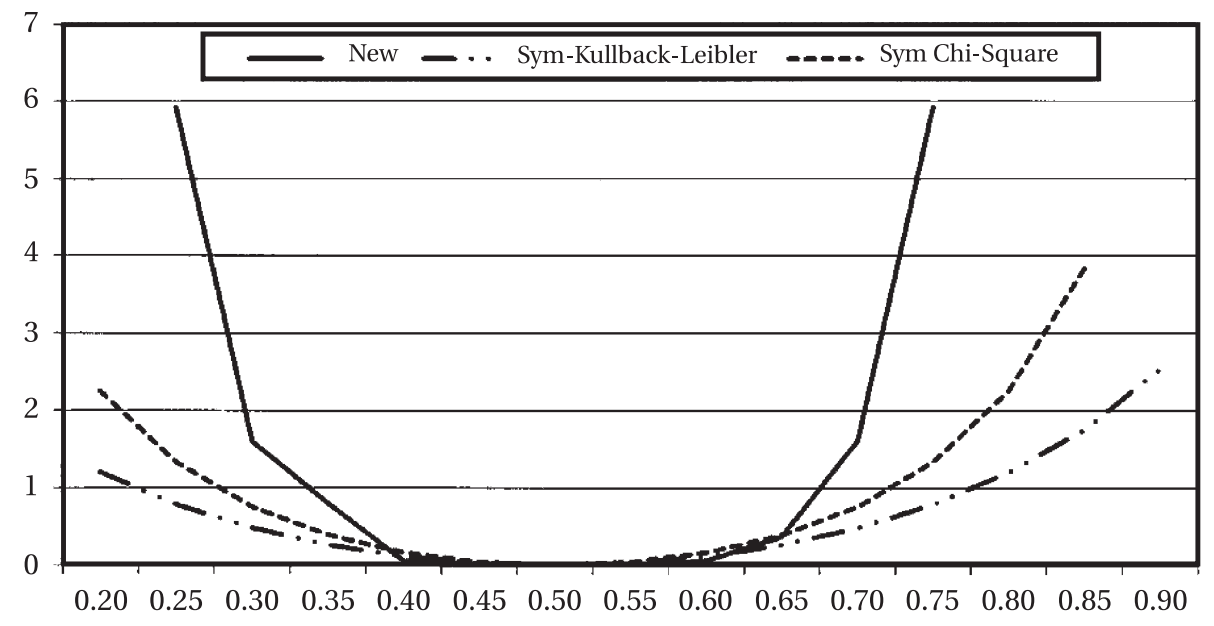

Figure 6.1: New $P^{*}(P, Q)$, Sym Chi-Square $\Psi(P, Q)$ and Sym Kullback - Leibler $J(P, Q)$.

Figure 6.1 shows the behaviour of $P^{*}(P, Q)(\mathrm{New}) \Psi(P, Q)$ [Sym-Chi-Square] and $J(P, Q)$ [Sym Kullback - Leibler]. We have considered $p=[a, 1-a)$ and $q=(1-a, a) a \in[0,1)$. It is clear from Figure 6.1 that the new measure has a steeper slope then $\Psi(P, Q)$ and $J(P, Q)$.

\section{References}

[1] S. M. Ali and S. D. Silvey, A general class of coefficients of divergence of one distribution from another, J. Roy. Statist. Soc. B, 28(1966), 131-142.

[2] J. Burbea and C. R. Rao, Entropy differential metric, distance and divergence measures in probability spaces: a unified approach, J. Multi. Analysis, 12(1982), 575-596.

[3] J. Burbea and C. R. Rao, On the convexity of some divergence measures based on entropy functions, IEEE Trans. on Infor. Theory, IT- 28(1982), 489-495.

[4] L. Csiszar, Information measures: A critical survey, In Trans. Seventh Prague Conf. On Information Theory, A, Academia, Prague (1967), 73-86.

[5] L. Csiszar, Information-type measure of difference of probability distributions and indirect observations, Studia Sci. Math. Hungar., 2(1974), 299-318.

[6] S. S. Dragomir, A converse inequality for the Csiszar's $\phi$ divergence, Inequalities for the Csiszar's $\phi f$ divergence in Information Theory, Chapter 1, Article 2 (Edited by S. S. Dragomir), http://rgmia.vu.edu.au/monographs/csiszar.htm

[7] S. S. Dragomir, Some inequalities for $(m, M)$-convex mappings and applications for the Csiszar's $\phi$ divergence, in Information theory, Inequalities for the Csiszar's $f$-divergence in Information Theory, (Edited by S. S. Dragomir), http://rgmia.vu . edu . au/monographs/csiszar.htm, 2000.

[8] S. S. Dragomir, Some inequalities for the Csiszar's $\phi$ divergence, Inequalities for the Csizar's $f$-divergence in Information Theory, (Edited by S. S. Dragomir), http://rgmia.vu.edu.au/monographs/ciszar.htm, 2000. 
[9] S. S. Dragomir, Upper and lower bounds for Csiszar's $f$-divergence in terms of the Kullback-Leibder distance and applications, Inequalities for the Csiszar's $f$-divergence in Information Theory, (Edited by S. S. Dragomir), http://rgrnia.vu.edu . au/monographs/ciszar.htm, 2000.

[10] S. S. Dragomir, Upper and lower bounds for Csiszar's $f$-divergence in terms of the Hellinger discrimination and applications, Inequalities for the Csiszar's $f$-divergence in Information Theory, (Edited by S. S. Dragomir), http://rgmia.vu.edu.au/monographs/csiszar.htm, 2000.

[11] S. S. Dragomir, V. Gluseceic and C. E. M. Pearce, Inequality Theory and Applications, (Edited by Y. L. Cho, J. K. Kim and S. S. Dragomir), Nova Science Publishes, Huntington, New York, 2001.

[12] S. S. Dragomir, S. Sunde and C. Buse, New inequalities for Jeffrey's divergence measures, Tamsui Oxford Journal of Mathematical Sciences, 16(2000), 295-309.

[13] K. Feretimos and T. Papaiopannou, New parametric measure of information, Information and Control, 51 (1981), 193-208.

[14] E. Hellinger, Neue Berunduring der Theorie der Quadratischen Formen von Un endlichen Vieden Veran derliehen, J. Reine Aug. Math., 136 (1909), 210-271.

[15] H. Jefferey, An invariant form for the prior probability in estimation problems, Proc. Roy. Soc. Lon., Ser. A, 186(1946), 453-461.

[16] R. A. Fisher, Theory of statistical estimation, Proe. Cambridge Philos. Soc., 22, 700-772.

[17] S. Kullback and A. S. Leibler, On information and sufficiency, Ann. Math. Stat., 22 (1951), 79-86

[18] P. Kumar and A. Johnson, On a symmetric divergence measure and information inequalities, Journal of Inequalities in Pure and Applied Mathematics, 16(2005), article 65.

[19] F. Osterreircher, Csiszar's f-divergences-Basic properties, Res. Report Collection http://rgmia.vu.edu.au/monographs/csiszar.htm, 2002.

[20] Renyi A., "On measures of entropy and information", In Proc. Fourth Berkeley Symp. on Math. Statist. And Prob., 1, University of California Press, Berkeley, CA, U.S.A (1961), 547-561.

[21] C. E. Shannon, A mathematical theory of communication, Bell Syst. Tech. Jour., 2 (1948), 623-659.

[22] R. Sibson, Information Radius, Z. Wahrsch. Verw. Gebiete, 14 (1969), 149-160

[23] L. J. Taneja, New developments in generalized information measures, Advances in Electronics and Electron Physics (Edited by P.W. Hawkes), 91 (1995), 37-135.

[24] L. J. Taneja and P. Kumar, Relative information of type-s, Csiszar's $f$-divergence and information inequalities, Information Sciences, 2003.

[25] L. J. Taneja, Relative Divergence Measures and Information inequalities, Inequality theory and applications, Vol. 4, Y. J. Cho., J. K. Kim and S. S. Dragomir (Eds.), Nova science Publishers, inc Huntington (2004), New York.

[26] L. J. Taneja, New developments in generalized information measures, Chapter in Advances in Imaging and Electron Physics, Ed. P.W. Hawkes, 91 (1995), 37-135.

[27] F. Topsoe, Res. Rep. Collection, RGMIA 2(1999), 85-98.

Department of Mathematics, Malviya National Institute of Technology, Jaipur-302017, Rajasthan, India.

E-mail: jainkc_2003@yahoo.co.in

Department of Mathematics, Malviya National Institute of Technology, Jaipur - 302017, Rajasthan, India.

E-mail: ruchimathur99@yahoo.com 